\title{
What's the proper policy?
}

T he semiannual inspection of animal housing and use areas at Great Eastern University went smoothly and the IACUC diligently followed up to assure that the few problems that were found had been corrected in the time frame set by the committee. The only remaining difficulty was with the Reinhold lab, where the inspectors had found surgical packs that had been sterilized a year ago by autoclaving but never re-sterilized. The IACUC considered this a potential threat to the health of the animals and therefore a significant deficiency. The committee informed the lab, verbally and in writing, that unused surgical packs must be re-sterilized every six months. However, Dr. Jan Reinhold responded that the policy of his lab was that sterilized but unopened and uncontaminated surgical packs could be kept indefinitely before use, as per published government guidelines ${ }^{1}$. The IACUC then gave Reinhold the written policy of the school's Department of Animal Resources, which was developed by that department's lab animal veterinarians and approved by the IACUC, and which clearly stated that unopened surgical packs must be re-sterilized every six months. After reading the policy Reinhold said that if Animal Resources wished to re-sterilize surgical packs every six months, that was fine with him; however, a policy that was approved by the IACUC for the Department of Animal Resources was not a general IACUC policy for the school and therefore it was not binding on his department or his laboratory. "Look," said Reinhold, "my lab keeps good medical records and your inspectors checked them and found no problems with surgical infections. And if there is scientific literature supporting our policy, why are you telling us we have to follow the policy of another department whose policy is based on opinion and nothing more."

The IACUC has many options to consider. How would you approach this issue?

\section{Jerald Silverman}

University of Massachusetts Medical School,

Worcester, Massachusetts, USA.

e-mail: Jerald.Silverman@umassmed.edu

Published online: 23 October 2018

https://doi.org/10.1038/s41684-018-0176-7

\section{References}

1. Rutala, W.A., Weber, D.J. and the Healthcare Infection Control Practices Advisory Committee (HICPAC). Guideline for disinfection and sterilization in healthcare facilities (2008). https://www.cdc.gov/infectioncontrol/guidelines/disinfection/

\section{Clear IACUC policies required}

\begin{abstract}
A $s$ the entity tasked with safeguarding animal welfare within the research setting, it is well within the IACUC's purview to ensure that all practices do not cause a threat to animal health. The inspectors should be commended for identifying this potential concern during the semi-annual inspection. However, rather than simply referring to a policy written by the Department of Animal Resources (DAR), the committee should be looking at what the IACUC policies are instead, as they require compliance from all animal users. The policy of the DAR does not extend beyond the DAR, unless other departments or laboratories choose to adopt it, or the IACUC mandates that all users have to follow it. Following Dr. Reinhold's comments, it will be good for the IACUC to take the opportunity to review/ consider the following:
\end{abstract}

(1) Is it necessary to re-sterilise unopened and uncontaminated surgical packs?

(2) Is there scientific literature to support this?

(3) Does the IACUC expect all animal users to adhere to one specific standard, i.e. is a policy required?

The CDC guidelines are based on multiple citations indicating that shelf life should be event-related, meaning that as long as the packaging of the sterile items is not compromised, re-sterilisation is not required. Other publications support the validity of this practice, for up to 96 weeks $^{1,2}$. Granted there are many factors that have to be considered in determining whether or not an item is compromised, e.g. material used for packaging, methods of packaging, how it has been handled, storage conditions (humidity, ventilation, presence of dust and/or vermin). Assuming that all these have been addressed, there seems to be little scientific evidence that unopened, uncontaminated surgical packs need an expiry date.

As such, the IACUC will first need to decide if re-sterilisation is necessary past a given time period, and/or under what circumstances. It may be beneficial to find out the reason why the DAR policy states that re-sterilisation is required every six months. Thereafter, an IACUC policy should be drafted and approved by the committee. The rationale for requiring re-sterilisation (or not) should be also conveyed. Once that is in effect, Dr. Reinhold will need to submit for an exemption and justify his position, if it is not in line with the IACUC policy. Apart from requesting scientific literature, one can also suggest that the sterility of the surgical packs be validated. Having records showing no surgical infections may not be sufficient, since subclinical infections may go undetected but still have significant impact on research outcomes, leading to poor science and a waste of animals. If Dr. Reinhold is able to demonstrate that his lab practices are able to keep surgical packs sterile, the IACUC can consider accepting this and grant Dr. Reinhold an exemption to the IACUC policy, if the IACUC decides on a fixed shelf life. However, this should be reviewed periodically to ensure that the lab practices remain the same.

Ultimately, the policies and recommendations that the IACUC provides should be based on scientific data and performance assessment. Periodic review of available literature and updating practices to current industry standards is important; the IACUC and DAR should routinely review their policies and standard operating procedures. As it stands, if Dr. Reinhold's surgical packs are indeed not open, and the IACUC is satisfied that the packs are not compromised, the IACUC may wish to reconsider enforcing the 6-month re-sterilisation requirement for Dr. Reinhold.

\section{Jassia Pang*, Michelle Loh, Yi Quan Tay, Yasmina Arditi Paramastri and Michele Marie Bailey \\ Comparative Medicine, National University of Singapore, Singapore, Singapore. \\ *e-mail: jpang@nus.edu.sg}

Published online: 23 October 2018 https://doi.org/10.1038/s41684-018-0177-6

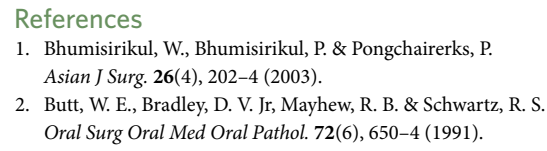

